\title{
Overshunting-associated myelopathy: report of 2 cases
}

\author{
Jason Man-kit Ho, MBChB, Hing-Yuen Law, FRCS(SN), Shing-Chau Yuen, FRCS, and \\ Kwong-Yui Yam, FRCS
}

\author{
Department of Neurosurgery, Tuen Mun Hospital, Tuen Mun, Hong Kong Special Administrative Region, China
}

\begin{abstract}
The authors present 2 cases of cervical myelopathy produced by engorged vertebral veins due to overshunting. Overshunting-associated myelopathy is a rare complication of CSF shunting. Coexisting cervical degenerative disc disease may further increase the difficulty of diagnosing the condition. Neurosurgeons and others who routinely evaluate patients with intracranial shunts should be familiar with this rare but possible diagnosis.
\end{abstract}

http://thejns.org/doi/abs/10.3171/2016.7.FOCUS16179

KEY WORDS overshunting; myelopathy; hydrocephalus; ventriculoperitoneal shunt

$\mathrm{O}$ VERSHUNTING-ASSOCIATED myelopathy is a rare complication of CSF shunting. ${ }^{4}$ Few case reports have been published over the years. Here we add 2 cases to the literature.

\section{Case Reports \\ Case 1}

History and Presentation

This 64-year-old woman had undergone ventriculoperitoneal (VP) shunt placement for treatment of hydrocephalus after clipping of a ruptured posterior communicating artery aneurysm. A medium-pressure Pudenz valve had been used. Fourteen years after the initial shunt surgery, the patient complained of progressive "stiffness of whole body." At that time she reported that she had experienced gait unsteadiness for 6 months and worsening dexterity of both upper limbs for 12 months.

Physical examination revealed a functioning shunt valve. The patient's distal upper-limb power was full but her proximal upper-limb power was Grade 4/5. All 4 limbs were spastic and intention tremor was observed. The supinator reflex was inverted bilaterally, and a Hoffman sign was noted on the left side. The patient's performance on the grip-and-release test (10 seconds test) was slow. Upper-limb proprioception was impaired. Light touch and temperature sensation were impaired on the right palm. The patient had an upgoing plantar reflex and ankle clonus bilaterally. Tandem walking could barely be performed.

T1-weighted Gd-enhanced MRI of the brain and cervical spine revealed diffuse pachymeningeal enhancement (Fig. 1 upper) and engorgement of vertebral veins from the $\mathrm{C}-1$ to the $\mathrm{C}-3$ level causing cord compression at the corresponding levels (Fig. 2A and B). T2-weighted imaging showed signal hyperintensity in the spinal cord (Fig. 3 left) at the C-1 level. Degenerative changes (marginal osteophytes and disc bulging) were noted at the C5-6 and C6-7 levels, with indentation of the anterior thecal sac.

\section{Operation and Postoperative Course}

Revision of the VP shunt was performed. The original medium-pressure Pudenz valve was changed to a Medtronic Strata II valve, with the valve set at position 1.0.

The patient's unsteadiness, numbness, and clumsiness improved within 1 week after shunt revision and had completely resolved 1 month after the operation.

An MRI examination performed 4 months postoperatively showed increased ventricular size when compared with preoperative imaging as well as resolution of pachymeningeal enhancement (Fig. 1 lower). The vertebral veins (Fig. 2C) were not engorged, and the spinal cord was well 

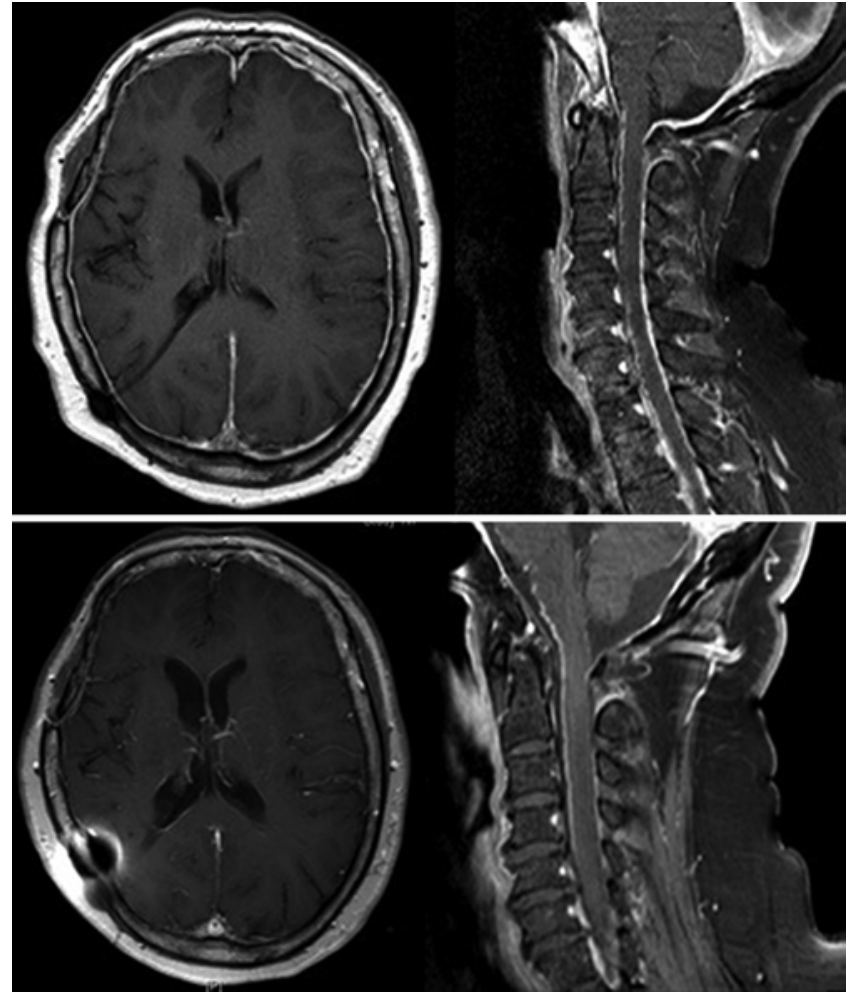

FIG. 1. Case 1. T1-weighted, Gd-enhanced MR images. Upper: Preoperative axial and sagittal images showing diffuse pachymeningeal enhancement. A right parietal ventricular shunt valve and catheter may also be seen. Lower: Postoperative axial and sagittal images obtained 4 months after shunt revision showing an increase in ventricle size and resolution of pachymeningeal enhancement.

decompressed. There was interval reduction of the T2 signal hyperintensity in the spinal cord at the $\mathrm{C} 1-2$ level of spinal cord (Fig. 3 right). The degenerative changes were static.

\section{Case 2}

History and Presentation

This 22-year-old woman had undergone insertion of a VP shunt at the age of 3 months for treatment of congenital hydrocephalus due to a posterior fossa arachnoid cyst. A partial cystectomy of the posterior fossa arachnoid cyst and placement of a cystoperitoneal shunt were performed following placement of the VP shunt. The patient underwent revision of the VP shunt (performed by a surgeon who was not affiliated with our institution) for suspected shunt blockage when she was 17 years old. When the patient presented to us, her shunt system had a mediumpressure Radionic valve. She complained of the feeling of "sunken eyes" and neck pain for 2 months with associated numbness of both palms and weakness of both hands. All limb reflexes were brisk. The patient's plantar reflex was downgoing bilaterally. The results of somatosensory evoked potential testing were normal.

Preoperative MRI of the brain (Fig. 4 left) showed diffuse pachymeningeal enhancement and small ventricle size, while MRI of the cervical spine (Figs. 5 and 6 upper) showed T2 signal hyperintensity and pachymeningeal enhancement of the spinal cord at the $\mathrm{C} 1-2$ level.

\section{Operation and Postoperative Course}

A Codman Hakim programmable valve with an antisiphon device was inserted with the initial pressure set at $8 \mathrm{~mm} \mathrm{Hg}$. The overshunting status was supported by the intraoperative finding of an opening pressure of the old Radionic valve of $2 \mathrm{~cm} \mathrm{H}_{2} \mathrm{O}$. The patient no longer had the feeling of "sunken eyes" at the 1-month follow-up visit, but her neck pain persisted, so the shunt setting was gradually increased. Ten months after shunt revision, the patient had headache with the valve pressure at $15 \mathrm{~mm} \mathrm{Hg}$, so it was lowered to $13 \mathrm{~mm} \mathrm{Hg}$.

At the follow-up evaluation 20 months after revision of the VP shunt, the patient no longer had headache, numbness, neck pain, or the sensation of "sunken eyes."

Postoperative MRI of the brain (Fig. 4 right) performed at 6 months after surgery showed interval increase in ventricle size and reduction in pachymeningeal enhancement, while MRI of the cervical spine (Fig. 6 lower) showed interval decrease in venous engorgement.

\section{Discussion}

Shunt overdrainage is seen in $10 \%-12 \%$ of patients with VP shunts. Manifestations include slit ventricle syndrome, intracranial hypotension syndrome (IHS), subdural fluid collections, and other rarer conditions..$^{10,12}$ The characteristic triad of IHS consists of postural headache, diffuse pachymeningeal enhancement on MR images obtained after gadolinium administration, and low CSF opening pressure. 1,9

Although orthostatic headache is the most characteristic symptom, patients may have chronic or intermittent headache or even no headache. ${ }^{7}$ The other common symptoms are anorexia, nausea, vomiting, dizziness, blurred vision, diplopia, tinnitus, ear fullness, facial numbness, and/ or neck pain; most of these symptoms are orthostatic in nature, while patients with overshunting-associated myelopathy may complain of limb numbness, weakness, and even gait disturbance. ${ }^{1,8,9}$

Diffuse pachymeningeal enhancement over the cerebral hemispheres on gadolinium-enhanced images was reported as the most characteristic neuroimaging feature of intracranial hypotension. However, cases without enhancement are also reported., ${ }^{3,7}$ This enhancement is attributed to dural venous dilation, which may extend to the pachymeninges of the posterior fossa and cervical spine. ${ }^{2}$ The other described MRI findings are engorgement of venous sinuses, subdural fluid collections, pituitary enlargement, and downward displacement of the brain. ${ }^{9}$ In a paper by Wolfe et al., ${ }^{13}$ the underlying pathophysiological mechanisms leading to spinal venous engorgement were well described. According to the Monro-Kellie doctrine, the sum of the intracranial blood and tissue volume remains constant. ${ }^{6}$ As long as the craniospinal system is intact, levels of all fluids, such as blood and CSF, are expected to remain stable. A deficit in one component will result in an excess of the others. In our cases, overshunting resulted in 

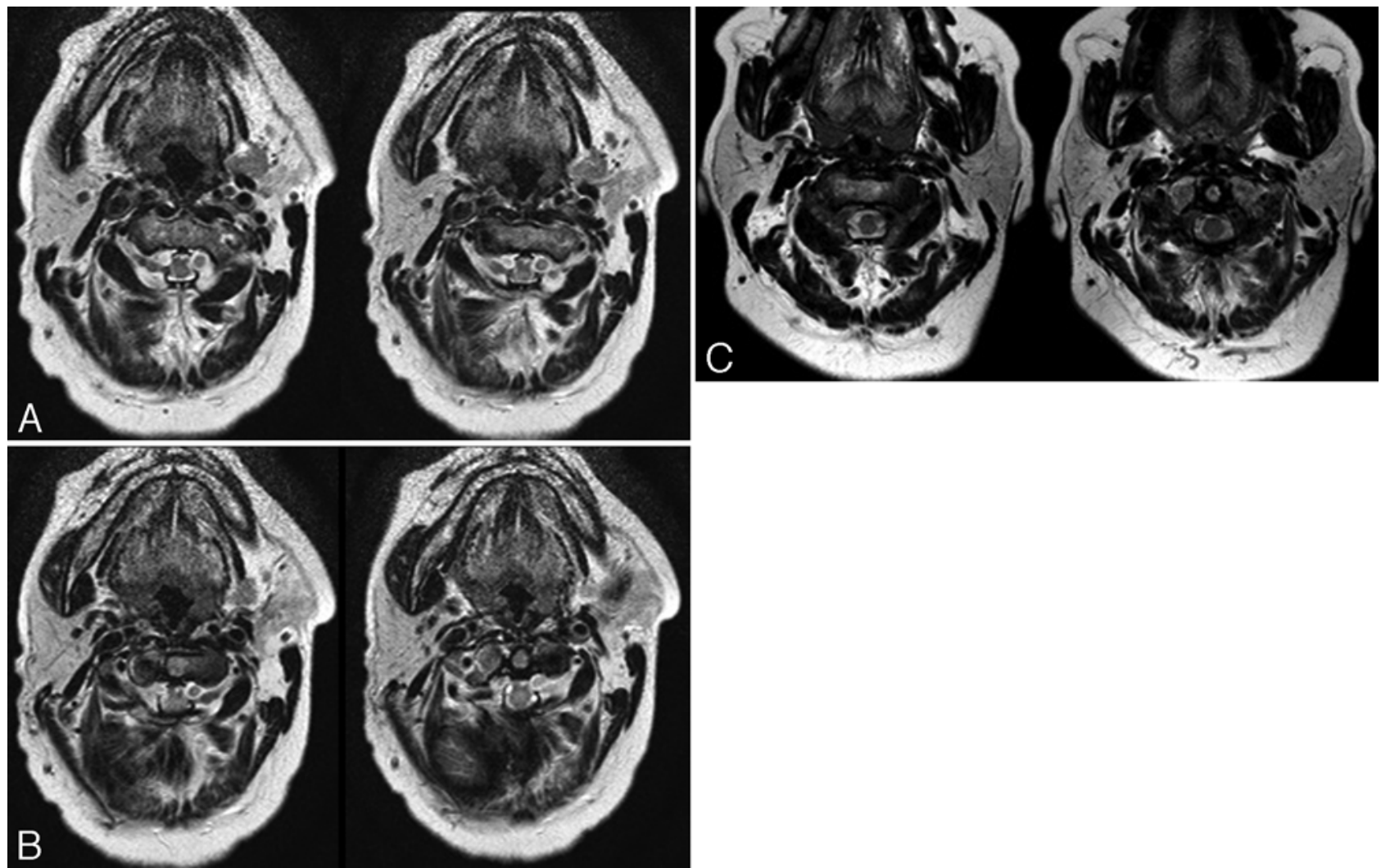

FIG. 2. Case 1. Axial T2-weighted MR images. A and B: Preoperative images obtained at the C-2 (A) and C-1 (B) levels showing vertebral vein engorgement and indented thecal sac and spinal cord. C: Postoperative images obtained at the C-1 level showing resolution of venous engorgement and relief of thecal sac and spinal cord compression 4 months after shunt revision.

engorgement of the vertebral veins and epidural venous plexus.

When venous engorgement becomes chronic, as reported by Mokri, ${ }^{5}$ fibrocollagenous proliferation contributes to dural thickening, which explains the spinal cord compression observed.
Patients with congenital or degenerative cervical spinal stenosis will be more susceptible to cord compression due to cervical engorgement of the veins. ${ }^{11}$

Additionally, the lack of awareness of this entity may lead to unnecessary treatments, for example, surgical decompression and fusion of the cervical spine. For patients

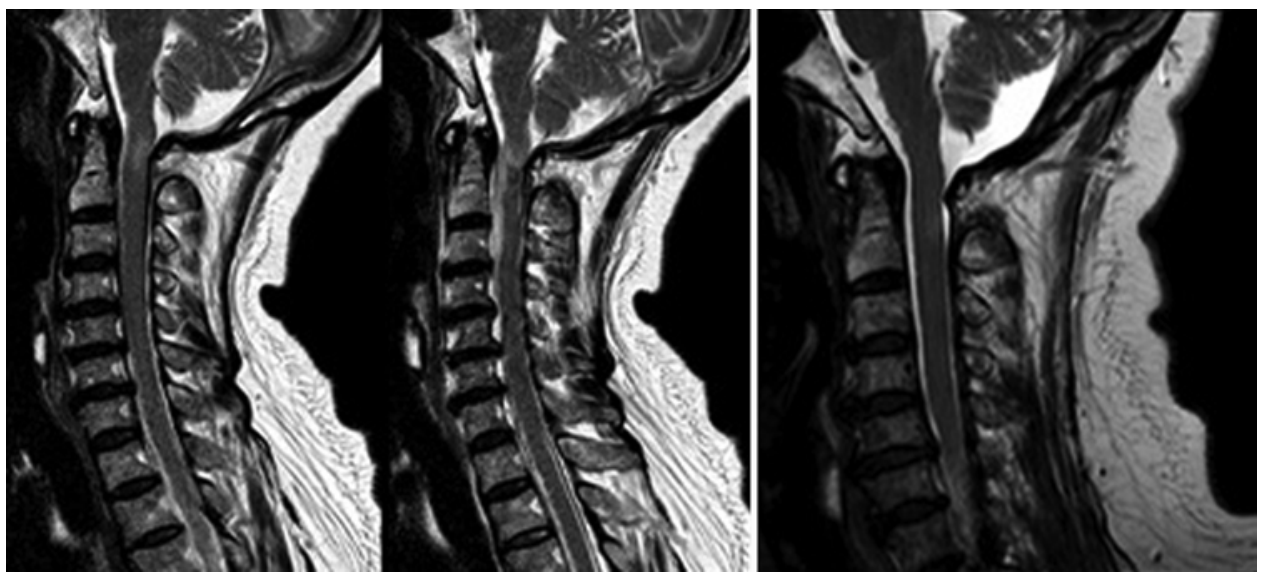

FIG. 3. Case 1. Sagittal T2-weighted MR images. Left: Preoperative images showing signal hyperintensity in the spinal cord at the $\mathrm{C}-1$ level and engorgement of the epidural venous plexus. Right: Postoperative image obtained 4 months after shunt revision showing minimal residual signal hyperintensity in the spinal cord at the C1-2 level. 

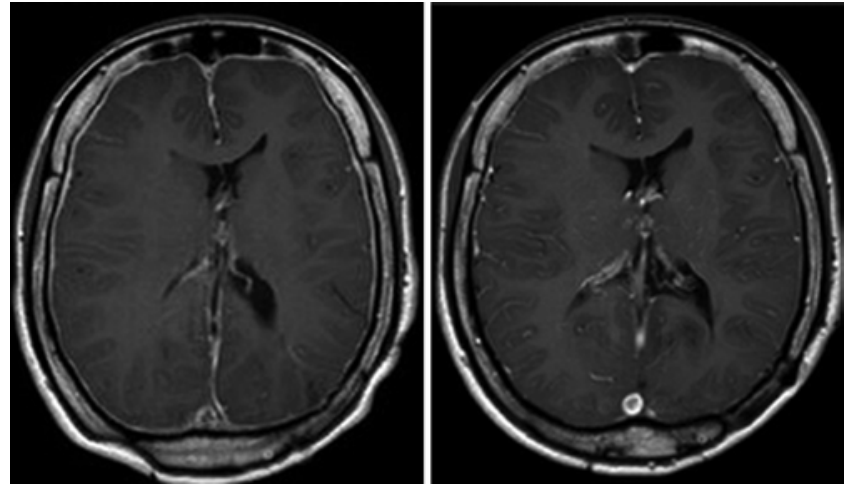

FIG. 4. Case 2. Contrast-enhanced T1-weighted axial MR images. Left: Preoperative image of the brain showing diffuse pachymeningeal enhancement and small ventricle size. Right: Postoperative image obtained 6 months after shunt revision showing a reduction in pachymeningeal enhancement and a slight increase in ventricle size.

with less florid signs, it is likely that the symptoms may even be attributed to psychosomatic causes, leading to treatment delay.

As the diagnosis of overshunting-associated myelopathy requires a high index of suspicion, it is easy for physicians and even patients themselves to overlook this potentially reversible problem. These patients might wrongly seek or be referred to orthopedic consultation, leading to delay in treatment. To detect this problem early and to provide appropriate and timely management, we recommend that all physicians who look after patients with shunts bear this condition in mind. Imaging of the brain for these patients, whether for diagnosis of overshunting or for evaluation of progress, should include lower cuts down to the cervicomedullary junction. Radiologists should also pay attention to and report any early signs of the development of overshunting. Patients could then be offered more frequent

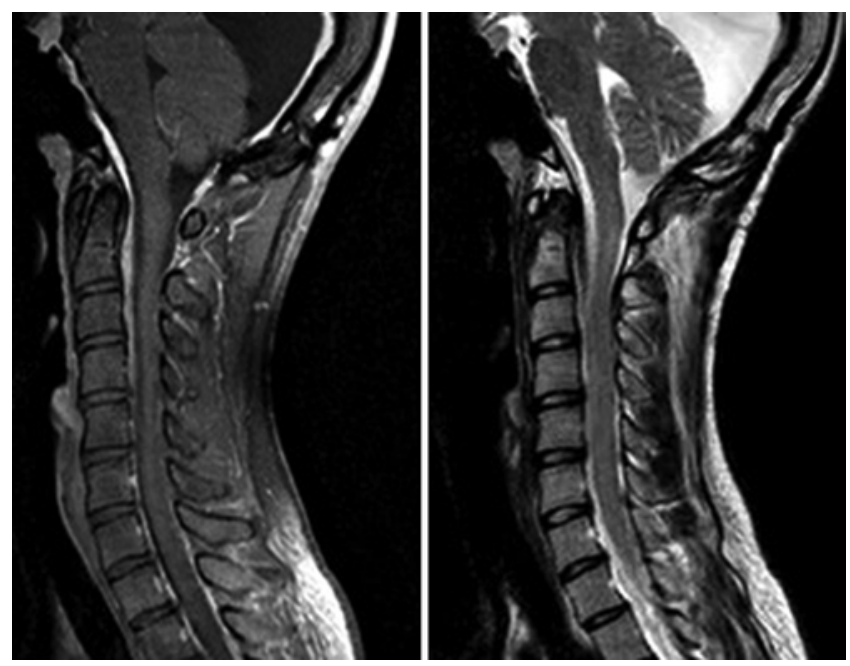

FIG. 5. Case 2. Sagittal T1-weighted Gd-enhanced (left) and T2weighted (right) MR images of the cervical spine showing, respectively, pachymeningeal enhancement and T2 signal hyperintensity in the spinal cord at the C1-2 level.

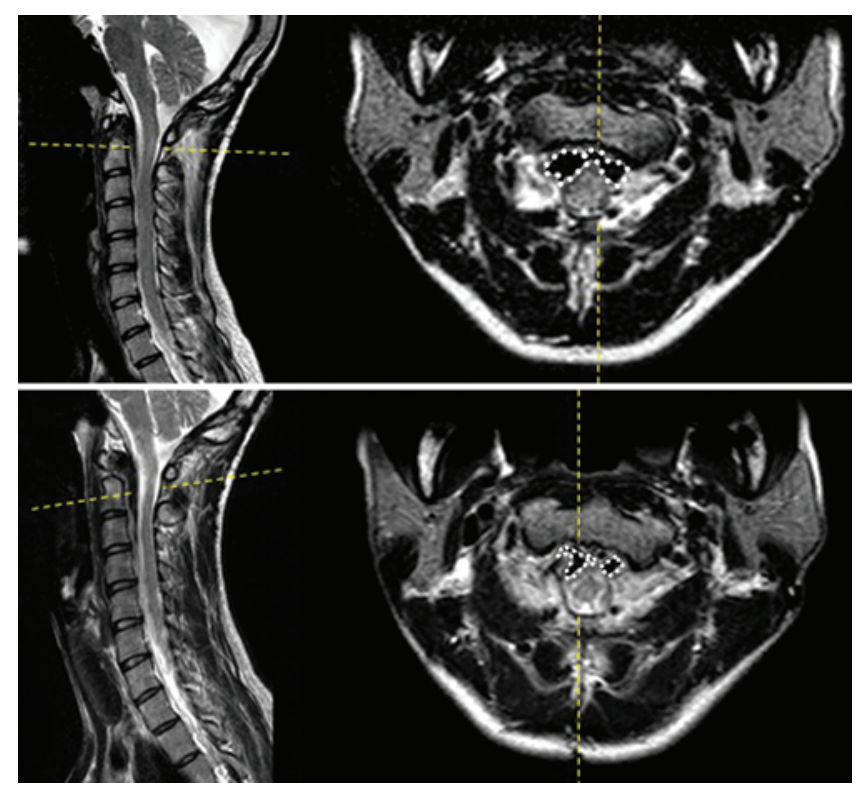

FIG. 6. Case 2. Preoperative (upper) and postoperative (lower) sagittal and axial T2-weighted MR images. The preoperative axial MR image shows vertebral venous engorgement (areas outlined in white dots) at the C-2 level where there was T2 signal intensity change. The corresponding postoperative image, which was obtained 6 months after shunt revision surgery, shows a reduction in the size of the area of signal hyperintensity as well as a reduction in venous engorgement.

follow-up for monitoring and early intervention to prevent the appearance of myelopathic symptoms. Whether programmable shunts should be used in all patients who need CSF shunting is debatable. But the use of a programmable shunt will allow easy correction of overshunting without requiring the patient to undergo another surgery to revise the shunt in case overshunting is encountered.

\section{Conclusions}

Although rare, chronic CSF overdrainage can lead to cervical myelopathy due to spinal cord compression by the dilated vertebral veins/epidural venous plexus. Awareness of this reversible condition can prevent unnecessary delay in treatment and thereby improve outcomes significantly.

\section{Acknowledgments}

We wish to acknowledge the assistance of the Department of Radiology and Nuclear Medicine, Tuen Mun Hospital, Hong Kong.

\section{References}

1. Chung SJ, Kim JS, Lee MC: Syndrome of cerebral spinal fluid hypovolemia: clinical and imaging features and outcome. Neurology 55:1321-1327, 2000

2. Fishman RA, Dillon WP: Dural enhancement and cerebral displacement secondary to intracranial hypotension. Neurology 43:609-611, 1993

3. Fuh JL, Wang SJ, Lai TH, Hseu SS: The timing of MRI determines the presence or absence of diffuse pachymeningeal enhancement in patients with spontaneous intracranial hypotension. Cephalalgia 28:318-322, 2008 
4. Howard BM, Sribnick EA, Dhall SS: Over-shunting associated myelopathy. J Clin Neurosci 21:2242-2244, 2014

5. Mokri B: Cerebrospinal fluid volume depletion and its emerging clinical/imaging syndromes. Neurosurg Focus 9(1):e6, 2000

6. Mokri B: The Monro-Kellie hypothesis: applications in CSF volume depletion. Neurology 56:1746-1748, 2001

7. Mokri B: Spontaneous cerebrospinal fluid leaks: from intracranial hypotension to cerebrospinal fluid hypovolemiaevolution of a concept. Mayo Clin Proc 74:1113-1123, 1999

8. Paldino M, Mogilner AY, Tenner MS: Intracranial hypotension syndrome: a comprehensive review. Neurosurg Focus 15(6):ECP2, 2003

9. Park ES, Kim E: Spontaneous intracranial hypotension: clinical presentation, imaging features and treatment. J Korean Neurosurg Soc 45:1-4, 2009

10. Pudenz RH, Foltz EL: Hydrocephalus: overdrainage by ventricular shunts. A review and recommendations. Surg Neurol 35:200-212, 1991

11. Ulrich NH, Maier M, Bernays RL, Krayenbuhl N, Kollias S: Cervical myelopathy due to chronic overshunting in a pediatric patient: case report and review of the literature. Turk Neurosurg 23:410-414, 2013

12. Weprin BE, Swift DM: Complications of ventricular shunts. Tech Neurosurg 7:224-242, 2002

13. Wolfe SQ, Bhatia S, Green B, Ragheb J: Engorged epidural venous plexus and cervical myelopathy due to cerebrospinal fluid overdrainage: a rare complication of ventricular shunts. Case report. J Neurosurg 106 (3 Suppl):227-231, 2007

\section{Disclosures}

The authors report no conflict of interest concerning the materials or methods used in this study or the findings specified in this paper.

\section{Author Contributions}

Conception and design: Law, Ho, Yuen. Acquisition of data: Law, Ho, Yuen. Analysis and interpretation of data: Law, Ho. Drafting the article: Law, Ho. Critically revising the article: all authors. Reviewed submitted version of manuscript: all authors. Approved the final version of the manuscript on behalf of all authors: Law. Administrative/technical/material support: Law, Ho, Yam. Study supervision: Yuen, Yam.

\section{Correspondence}

Hing-Yuen Law, Department of Neurosurgery, Tuen Mun Hospital, Tuen Mun, Hong Kong Special Administrative Region, China.email: 1hy622@ha.org.hk. 